\title{
Design and Testing of a Fluid-Cable Transmission
}

\author{
$1^{\text {st }}$ Jorge Zapote Castilla \\ Dept. Mechanical Engg \\ University of Calgary \\ Calgary, Canada \\ jbzapote@ucalgary.ca
}

\author{
$2^{\text {nd }}$ Alexander Irwin \\ Dept. Mechanical Engg \\ University of Calgary \\ Calgary, Canada \\ alexander.irwin@ucalgary.ca
}

\author{
$3^{\text {rd }}$ Peter Goldsmith \\ Dept. Mechanical Engg \\ University of Calgary \\ Calgary, Canada \\ peter.goldsmith@ucalgary.ca
}

\author{
$4^{\text {th }}$ Robert Brennan \\ Dept. Mechanical Engg \\ University of Calgary \\ Calgary, Canada \\ rbrennan@ucalgary.ca
}

\begin{abstract}
This paper describes the principles, design, and testing of a novel transmission that combines the advantages of fluid and cable elements. The cable and fluid run concentrically inside a single flexible hose to connect a master piston to a remote slave piston. Together, the fluid and cable transmit bidirectional forces and motions. Since fluid acts on only one side of each piston, low-friction, leak-proof rolling diaphragms may be used instead of o-rings. The fluid-cable transmission eliminates the need for antagonistic cables and overcomes the friction and force limitations of conventional push-pull cables. It may be used as a low-friction remote actuation system in medical devices, assistive technology, and robotic manipulators. We analyze its transmission stiffness, describe its design and manufacture, and report on progress towards comparative testing.

Index Terms-remote actuation system, transmission design, haptic transparency, telerobotics
\end{abstract}

\section{INTRODUCTION}

Remote actuation systems (RAS) use stationary motors to drive the moving joints of robots and other mechanisms so that the weight and inertia of the motors are not borne at the joints. Much research on RAS is aimed at wearable assistive devices [1] to eliminate the weight of motors on the limbs of users. Some of these RAS are pneumatic transmissions [2], [3], but most are based on Bowden cables [1], [4], [5].

In [6], a rolling cable transmission is proposed that allows articulation of straight cable segments in a cable RAS, thus reducing Bowden cable friction [7], [8]. However, the transmission requires gears between the segments and is complicated by the need to run the two antagonistic cables in separate tubes, which also add bulk.

Rolling diaphragm cylinders are used in [9] to develop a low-friction fluid transmission based on antagonistic pairs of cylinders. The authors use these transmissions in [10] to remotely actuate the joints of a two-armed humanoid robot that is slave to an identical master robot. The lowfriction transmissions provide high haptic transparency, which cannot be attained by conventional hydraulics due to high friction seals [11]. In [12], a long-stroke rolling diaphragm cylinder was developed to increase range of motion. In [13], a 'constant-force' spring was used to precompress the fluid and eliminate one of the antagonist cylinders, but the spring was bulky and had nonzero stiffness.

Besides assistive devices, low-friction RAS have applications in medical devices. A hand-controlled cable-driven robot

This research was funded by NSERC for minimally invasive surgery is described in [14]. RAS can also be used in medical telerobotics, where the main focus is telerobotic surgery [15]. However, RAS-based telerobots can also be used for other medical procedures. Cable-driven robots for teleoperated ultrasound are described in [16]-[19].

Currently, physicians use teleconferencing technology to examine, diagnose, and advise remote patients. Telerobotics would add significant telepresence to telemedicine by allowing physicians to interact physically with remote patients. A doctor could use a remote robot to palpate tissue, position a patient's limbs, and manipulate medical devices and probes to acquire detailed diagnostic information, such as close-up views and internal images. Such interaction requires lightweight, human-safe robots with low-friction drives that can transmit forces between the doctor and patient with high haptic transparency.

This paper describes the design and testing of a novel RAS that addresses some limitations of existing systems. While our target application is high-fidelity telerobotics for enhanced telemedicine, the proposed RAS may be used in any application where a compact, low-friction transmission is needed.

\section{The Fluid-CABLE TRANSMission}

We propose a novel RAS that combines the benefits of fluid and cable into a single transmission, called a fluid-cable transmission, or FCT. In this section, we describe the basic invention, for which a patent is pending [20].

The simplest FCT configuration is shown in Figure 1. A stationary master cylinder is connected to a moving slave cylinder by a flexible hose that contains hydraulic fluid and a concentric wire cable that joins the master and slave pistons. The fluid transmits compressive (pushing) forces from master to slave, while the wire cable transmits tensile (pulling) forces. The hose also serves as a lubricated cable housing that bears compressive forces to oppose the cable tension, like the brake and gear (Bowden) cables on a bicycle.

Since the hose and cable are flexible, the slave cylinder is mobile and thus can actuate a moving mechanism, such as a gripper, tool, or robot joint. Very light-weight robot arms can be designed using FCTs, as the robot does not need to carry the weight of its motors, which may be housed together with the master cylinders in a stationary base. 


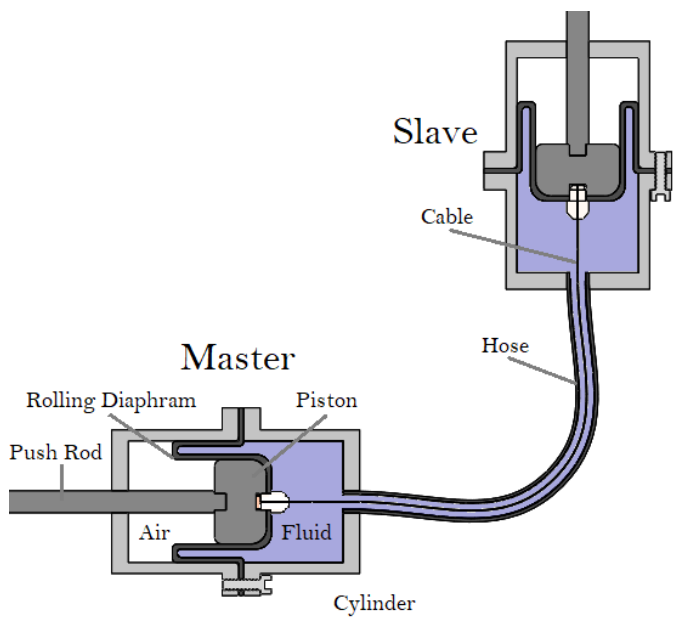

Fig. 1. Fluid-Cable Transmission (FCT)

Since the wire cable in the FCT can transmit tensile forces, fluid is only required on one side of the pistons, whereas conventional hydraulic transmissions require fluid on both sides each piston (and an additional connecting hose) to allow bidirectional motion and forces. Conventional hydraulic pistons require o-ring seals, which introduce significant friction and may leak. In contrast, FCT pistons can be sealed by lowfriction, leak-proof rolling diaphragms because the pressure gradient across each piston never reverses (which would invert the diaphragm). Also, no seal is needed where the push rods exit each cylinder, as there is no fluid on that side.

The FCT also has advantages over cable drives. First, since cables can only pull, each joint of a cable-driven robot arm requires a pair of antagonistic cables, which are difficult to route to the multiple moving joints of a robot arm, whereas an FCT requires just one cable. Second, an FCT can provide much higher transmission stiffness (and hence positional accuracy) than a cable drive. This is achieved by pretensioning the FCT wire cable to keep the fluid in compression. If an incompressible liquid is used as the fluid, the slave piston of an FCT tracks the master piston exactly, whereas cable deformation in a cable drive introduces tracking errors. A detailed stiffness analysis is presented in the next section.

Friction between the wire cable and the FCT hose in Figure 1 can be eliminated by using pulley joints to connect straight sections of hose, as shown in Figure 2. The wire passes around a pulley that is immersed in the fluid between two hose sections, preventing the wire from contacting the inner surface of the hose. If a hydraulic swivel joint is used to connect the master hose to the pulley housing, then the slave and pulley can rotate freely about the axis of the master hose.

Mounting the pulley housing and slave cylinder on the same rigid link eliminates axial compression in the slave hose. As described in Section IV, additional pulleys and swivel joints can give the slave full (6 DOF) mobility without hose bending, thereby eliminating cable/hose friction.

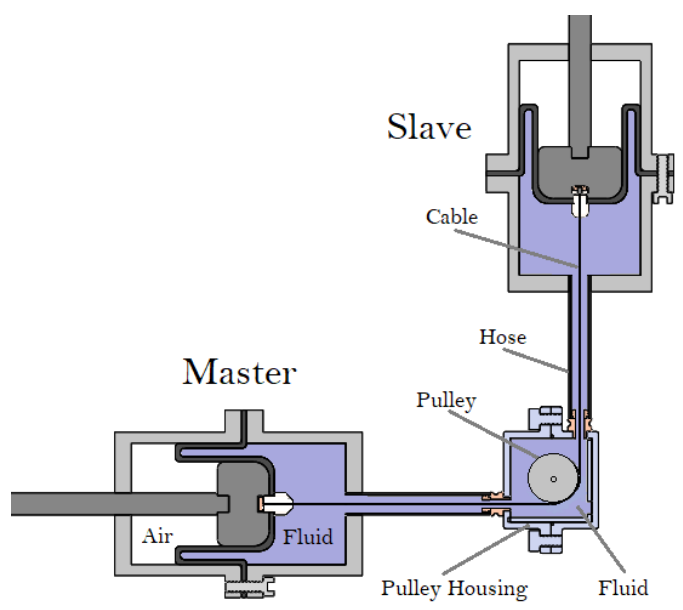

Fig. 2. FCT with Pulley

\section{FCT STIFFNESS ANALYSIS}

\section{A. FCT with Liquid as Fluid}

We first analyze the case when the FCT fluid is a liquid (versus gas). Since the wire cable in the FCT provides tensile forces, one might expect that the FCT stiffness will be lower in tension than compression. However, this is not the case.

The true purpose of the cable is to keep the fluid in compression. This is achieved by pretensioning the cable against the fluid at a tension of $p$, chosen to exceed the maximum external tensile load that might be applied to the slave push rod. Suppose that the system is in static equilibrium with an external tensile load $f$ (negative if compressive) applied to the slave push rod. Assume that friction is negligible and the master cylinder is held in place by an equal force $f$ acting on the master push rod. Let $f_{c}>0$ be the tension in the wire cable, and let $f_{f}=P A>0$ be the compressive force applied by the fluid to the slave piston, where $P$ is the fluid pressure and $A$ is the cross-sectional area of the cylinder. Then static equilibrium of the slave piston implies that

$$
f=f_{c}-f_{f} .
$$

When there is no external load, $f=0$ in (1) gives $f_{c}=f_{f}=p$, and the pretension of the wire equals the precompression of the fluid. This pretension stretches the cable and compresses the fluid. Let $x$ be the displacement of the slave piston (upward in Figure 1) due to the applied load $f$ (i.e. $x=0$ when $f=0$ ). Since the master piston is fixed, this is due to further stretching of the wire and (to a lesser extent) decompression of the fluid.

Assuming that the wire is linearly elastic, the displacement $x$ adds a force of $k_{c} x$, to the tension $f_{c}$ in the wire cable, where $k_{c}$ is the cable stiffness:

$$
f_{c}=p+k_{c} x .
$$

Similarly, if $k_{f}$ is the stiffness of the fluid, then the 
displacement $x$ reduces the compressive force in the fluid to

$$
f_{f}=p-k_{f} x .
$$

Substituting (2) and (3) into (1) gives

$$
\begin{aligned}
f & =\left(p+k_{c} x\right)-\left(p-k_{f} x\right) \\
& =\left(k_{c}+k_{f}\right) x,
\end{aligned}
$$

which shows that the transmission stiffness is the sum of the cable and fluid stiffness. This is not surprising, as the cable and fluid act in parallel. Thus, the effect of the pretension is to create a hybrid fluid/cable that has a stiffness of $k_{c}+k_{f}$, regardless of whether the external load $f$ is positive (tensile) or negative (compressive).

In the case of a liquid fluid, typically $k_{f}>>k_{c}$, so (5) gives $f \approx k_{f} x$. In this case, the liquid force adjusts to match the external force while the cable force stays nearly constant. Also, for compressive loads $(f<0)$, (5) remains valid as long as the magnitude of $f$ does not exceed $\left(k_{f} / k_{c}\right) p>>$ $p$. In conclusion, when liquid is used as the fluid, the FCT provides much higher transmission stiffness than a cable drive, regardless of the load direction.

\section{B. FCT with Gas as Fluid}

When the fluid is a gas, the analysis is nearly identical to that in the previous section, except that $p$ must be chosen to exceed the maximum external compressive load expected. The same result (5) is obtained, but now typically $k_{f}>>k_{c}$, so (5) gives $f \approx k_{c} x$. In this case, the cable force adjusts to match the external force while the gas force stays nearly constant. For tensile loads $(f>0)$, (5) remains valid as long as the magnitude of $f$ does not exceed $f<\left(k_{c} / k_{f}\right) p$, which is larger than $p$.

In conclusion, when gas is used as the fluid, the FCT provides much higher transmission stiffness than a pneumatic drive, regardless of load direction. Pneumatic drives cannot provide precise position control because gases are too compressible. A pneumatic FCT overcomes this because it increases the transmission stiffness to that of a cable, while using a lightweight gas to maintain the cable tension.

\section{Articulation}

Figure 3 shows two segments of cable hose connected by two pulley units of the type shown in Figure 2. The two pulley housings are connected by a swivel joint that allows rotation between the two segments about a perpendicular axis. An additional swivel joint where the vertical hose enters its pulley housing adds a second DOF, resulting in a 2-DOF spherical joint. Three such pairs connected in series by four tube segments gives full 6-DOF mobility of the slave cylinder with respect to the master cylinder.

\section{Rolling Diaphragm Design and Testing}

\section{A. Diaphragm Design}

As explained in Section II, an advantage of the FCT over conventional hydraulics is that o-ring seals may be replaced by low-friction (and leak-proof) rolling diaphragms, which have

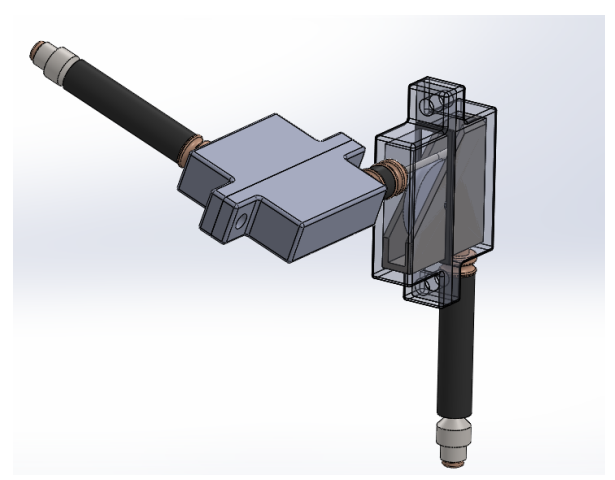

Fig. 3. Fluid-Cable Swivel Joint

been shown to have friction comparable to gap seals [12]. Initial designs for the rolling diaphragm were analyzed by FEA simulations to determine strain due to loading.

Figure 4 shows the strain in the diaphragm under fluid pressure. The diaphragm position and orientation corresponds to that of the master cylinder shown in Figure 2. The diaphragm is clamped at its flange, supported along A by the cylinder, and clamped at $\mathrm{C}$ (representing the piston). Fluid pressure of $125 \mathrm{psi}$ is applied to the right-hand side of the diaphragm as in Figure 2, filling the rolled region between piston and cylinder.

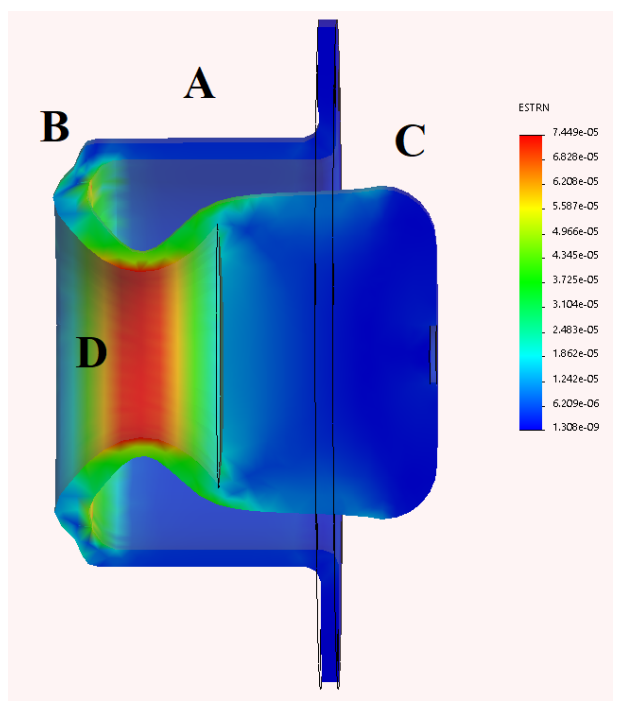

Fig. 4. Diaphragm Strain

The location of maximum strain is at $\mathrm{B}$ and $\mathrm{D}$, where the diaphragm bends; this is the critical strain for determining sufficient diaphragm thickness. The length and shape of the high-strain region depends on the piston length and position. A shorter piston provides a greater stroke but results in higher strains in the diaphragm.

\section{B. Diaphragm Manufacture}

Based on the success of dip coating for the manufacture of diaphragms in [12], this was first process we investigated, using Rebound 25 silicone by Smooth On Inc. While the 
method achieved full coverage of the mold, the diaphragm thicknesses produced were too thin for our requirements. Repeated dipping increased the material thickness, but this lengthened the process and produced imperfections. Figure 5 shows surface artifacts resulting from repetitive dip coating, which may weaken the diaphragm and affect its rolling motion.

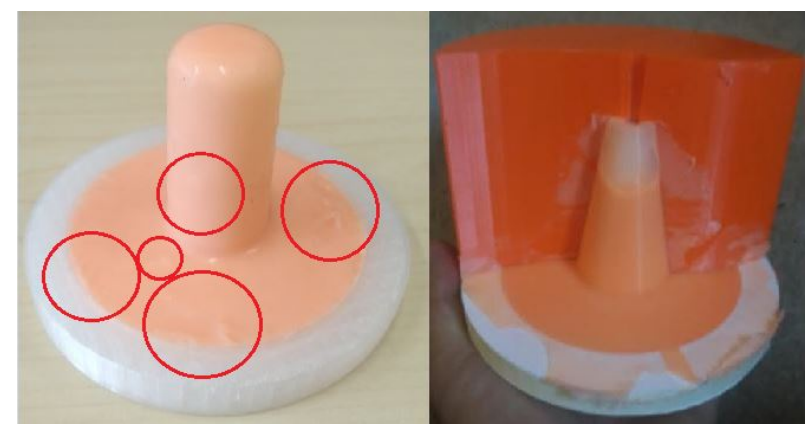

Fig. 5. Dip Coating vs. Injection Molding

The second process investigated was low pressure injection molding using a large volume syringe. The right image of Figure 5 shows that injection molding improved surface finish and uniformity of thickness but initially produced a mold void at the top of the mold. Redesign of the mold shape eliminated this problem resulting in the finished diaphragm of Figure 6, which has uniform thickness and a smooth surface finish.

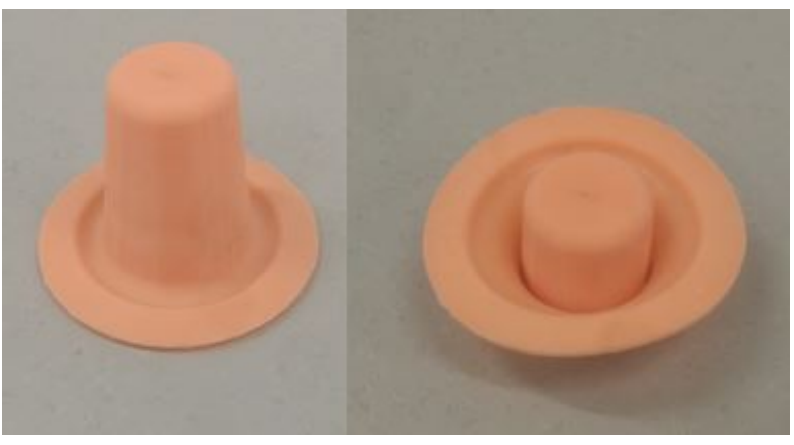

Fig. 6. Injection Molded/Cast Diaphragm

Besides the dip coating and injection molding methods, we also experimented with spray deposition of rubber. However, this approach was not effective due to thin layer thickness, drying time, and non-elastic behaviour of the finished diaphragm.

\section{Cylinder Design}

The first prototypes of the FCT system were designed to be easily manufactured, assembled and operated. Components were designed larger than necessary to simplify assembly. They were also designed to be easily 3D printed and/or CNC machined. Figure 7 shows a single cylinder in an exploded view. It consists of the two halves of the cylinders, the piston, rolling diaphragm and accompanying fasteners and hydraulic fittings.

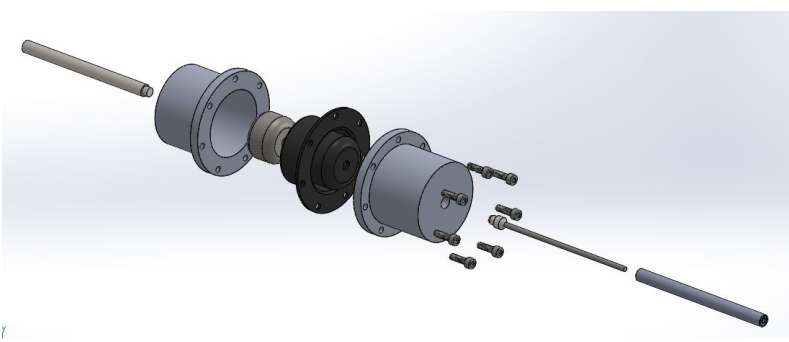

Fig. 7. Cylinder Exploded View

\section{Cylinder Manufacture}

Figure 8 shows a prototype of the complete FCT. The bolted flange in the blue cylinder was simplified in a subsequent prototype (red cylinder), which uses a threaded interface instead for ease of assembly. The cylinders were 3D printed for rapid validation. The stereolithography files were sliced, converted to G-code and printed on a Prusa mk3 fused deposition machine using standard $1.75 \mathrm{~mm}$ PLA filament.

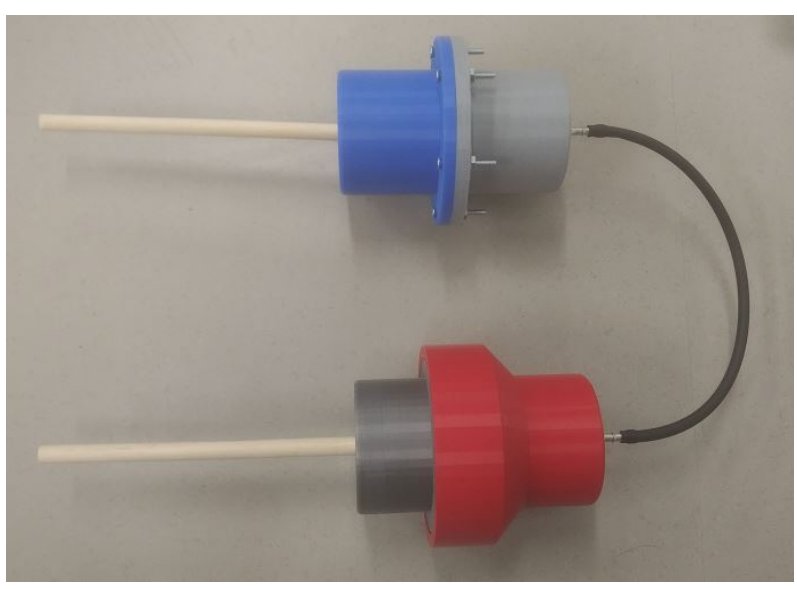

Fig. 8. Printed FCT Prototype

Initial qualitative testing yielded the following observations:

1) Positive pressure in the fluid cavity increases the quality of the rolling action of the diaphragms;

2) Threading the piston-cable anchor connection reduces the risk of leaking.

We are developing higher quality $\mathrm{CNC}$ machined prototypes using CAM programs to generate components. A clear acrylic cylinder prototype is also being developed to observe the rolling action of diaphragms within the cylinders.

\section{RAS TEST BED}

\section{A. Purpose}

We designed a test bed to compare the performance of FCT prototypes with that of other remote actuation systems, such as push-pull cables, cable drives, and master-slave hydraulics. The purpose is to assess whether the FCT is more suitable than other RAS for use in human-robot systems, such as those described in Section I. Much of the research on human-robot 
systems focuses on higher-level system design, with comparatively little attention paid to the choice of transmission. Studies that do consider transmission design typically test one transmission type with no comparative testing [21].

We designed a novel testing apparatus that allows a human operator to perform a remote task using interchangeable RAS that can be compared. The RAS transfers an operator's hand motions from an input device to an output device to perform a remote task. Operators can compare different RAS and assess their haptic transparency, i.e. how well the operator feels remote forces. The accuracy of performed tasks can also be analyzed and compared for different RAS.

\section{B. Design Requirements}

Key design parameters include the number of degrees of freedom (DOF), the simulated task, and mechanical design considerations.

1) Degrees of Freedom: The testing system was chosen to have two DOF. Although a 1-DOF system would be simpler, a 2-DOF system allows us to consider more complex (and hence more realistic) tasks. Also, how well the operator can coordinate the 2-DOF to complete a task provides a measure of accuracy and haptic transparency. A system with more than two DOF would be more complicated than necessary for testing purposes. For example, a 3-DOF system would require three copies of the RAS being tested, increasing the time and cost to manufacture each transmission system to be tested and the time to swap out RAS between tests, without significantly improving the usefulness of test results.

2) Task Selection: A drawing task was selected, where the operator traces a drawing at the input device to reproduce the drawing at the output device. This makes the testing device planar and gives a direct sense of system accuracy by comparing the input drawing to the the output drawing. It also places haptic transparency in the context of a familiar task.

3) Mechanical Design: The key mechanical consideration is the kinematics of the master-slave plotting mechanism. Typical plotting mechanisms utilize a rectangular frame and serial actuation methods. A triangular frame and parallel link design was chosen instead for both the input and output device. This results in a more rigid truss-like structure so that the transmission stiffness between the input and output is determined by the RAS being tested rather than the stiffness of the test apparatus. Also, since the FCT transfers translational (versus rotational) motion, translational (i.e. prismatic) joints are actuated in each device. Finally, a parallel-link design requires less movement of the master and slave cylinders, which need only accommodate passive rotational motion.

\section{Apparatus Design}

Based on the above requirements, we developed a design consisting of two triangular pen-plotters linked by two copies of the RAS being tested, as shown in Figures 9 and 10. The green master cylinder of the input device connects to the green slave cylinder of the output device, and similarly for the red master-slave pair. A human subject operates a pen at the apex of the input device to trace a drawing, and this motion is transferred by the two RAS to the output device, which plots the image on a transparent sheet that may be overlaid on the input image to assess accuracy.

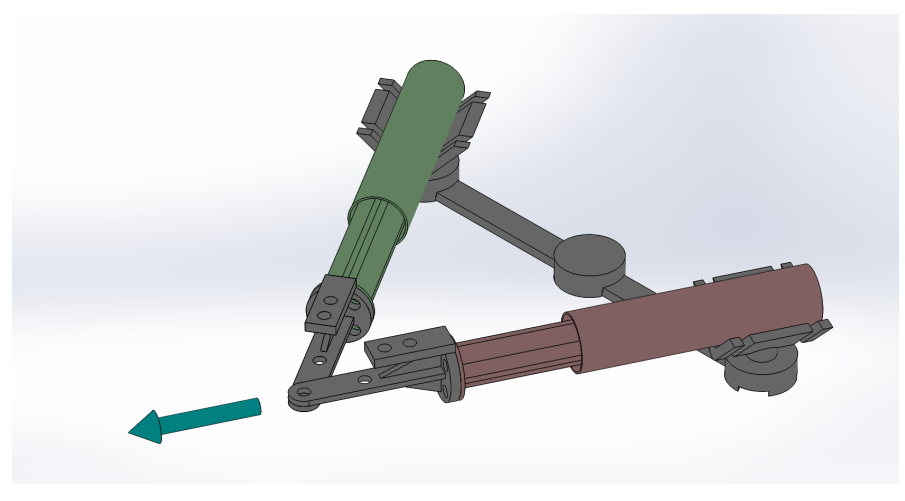

Fig. 9. Input Device

Extension of the master piston in each RAS results in contraction of the slave piston, as shown by the arrows in Figures 9 and 10. Thus, the actuation direction of each RAS must be reversed at the output device to correctly map input motions to output motions. This reversal is accomplished by the inclusion of extension links connected to the slave rods on the output device, as shown in Figure 10.

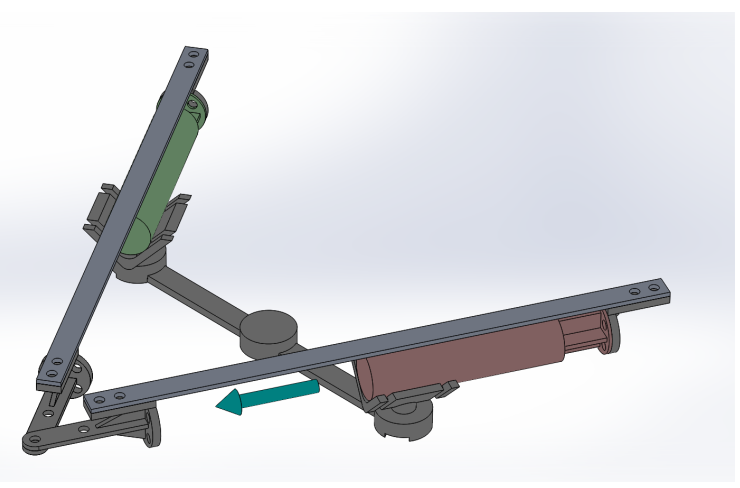

Fig. 10. Output Device

To ease the process of changing the RAS being tested, the pivot joints of the plotter are designed to accommodate a variety of cylinder diameters by using an open-angle mount combined with use of cable ties or pipe clamps to secure the transmission to the pivot joint, as shown in Figure 11. This allows several types and sizes of transmission to be tested.

We chose to manufacture most components of the prototype apparatus via 3D-printing as described in Section V-D. However, the extension link will be manufactured from stock aluminum parts to achieve the strength and stiffness required.

\section{CONCLUSIONS AND FUture WORK}

We introduced a novel remote actuation system (RAS) called the Fluid-Cable Transmission (FCT), which is essentially a hybrid push-pull cable composed of wire and fluid. The 


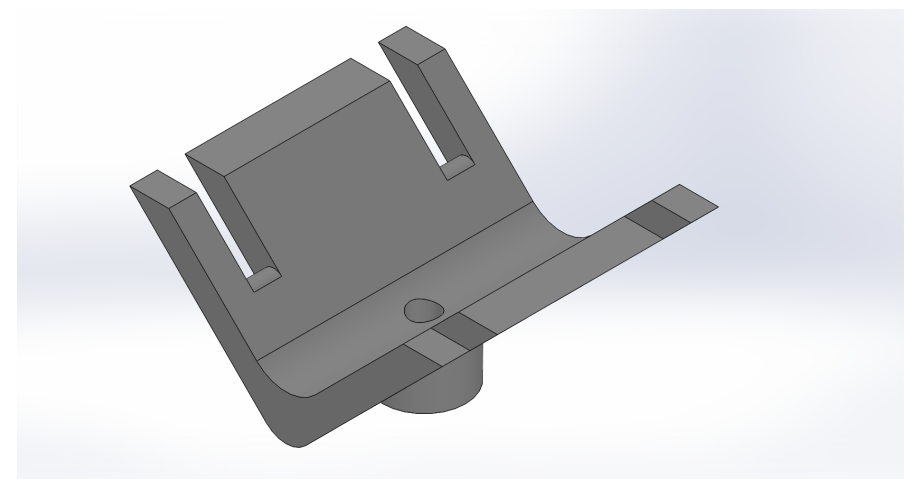

Fig. 11. The Pivot Joint

wire provides tension, while the fluid provides compression. If the wire is preloaded against the fluid, the transmission stiffness is the sum of the wire stiffness and the fluid stiffness, regardless of the external load direction. Hence, a liquid FCT is much stiffer than a cable drive, and a pneumatic FCT is much stiffer than a pneumatic drive.

The wire cable also results in fluid on only one side of the piston, allowing low-friction rolling diaphragm seals to be used in place of high-friction o-rings. Dip coating, injection molding, and spray coating methods were investigated for the manufacture of the diaphragms, with injection molding found to be the most effective. While this led to rapid prototyping of the diaphragm, we will also investigate press forming of a thermoplastic elastomer, as this method is used in industry.

A complete FCT prototype was designed and built, and a test apparatus was designed for investigating the performance of the FCT compared to other RAS. Results of this testing will be reported in future work.

\section{REFERENCES}

[1] RARC Gopura, DSV Bandara, Kazuo Kiguchi, and George KI Mann. Developments in hardware systems of active upper-limb exoskeleton robots: A review. Robotics and Autonomous Systems, 75:203-220, 2016.

[2] Ricardo Morales, Francisco Javier Badesa, Nicolás García-Aracil, José María Sabater, and Carlos Pérez-Vidal. Pneumatic robotic systems for upper limb rehabilitation. Medical \& biological engineering \& computing, 49(10):1145, 2011.

[3] Guido Belforte, Laura Gastaldi, and Massimo Sorli. Pneumatic active gait orthosis. Mechatronics, 11(3):301-323, 2001.

[4] Urs AT Hofmann, Tobias Bützer, Olivier Lambercy, and Roger Gassert. Design and evaluation of a bowden-cable-based remote actuation system for wearable robotics. IEEE Robotics and Automation Letters, 3(3):2101-2108, 2018.

[5] Christopher J Nycz, Tobias Bützer, Olivier Lambercy, Jumpei Arata, Gregory S Fischer, and Roger Gassert. Design and characterization of a lightweight and fully portable remote actuation system for use with a hand exoskeleton. IEEE Robotics and Automation Letters, 1(2):976-983, 2016.

[6] Jiun-Yih Kuan, Kenneth A Pasch, and Hugh M Herr. A highperformance cable-drive module for the development of wearable devices. IEEE/ASME Transactions on Mechatronics, 23(3):1238-1248, 2018.

[7] Qingcong Wu, Xingsong Wang, Lin Chen, and Fengpo Du. Transmission model and compensation control of double-tendon-sheath actuation system. IEEE Transactions on Industrial Electronics, 62(3):1599-1609, 2015.
[8] V. Agrawal, W. J. Peine, and B. Yao. Modeling of transmission characteristics across a cable-conduit system. IEEE Transactions on Robotics, 26(5):914-924, Oct 2010.

[9] John P Whitney, Matthew F Glisson, Eric L Brockmeyer, and Jessica K Hodgins. A low-friction passive fluid transmission and fluid-tendon soft actuator. In Intelligent Robots and Systems (IROS 2014), 2014 IEEE/RSJ International Conference on, pages 2801-2808. IEEE, 2014.

[10] John P Whitney, Tianyao Chen, John Mars, and Jessica K Hodgins. A hybrid hydrostatic transmission and human-safe haptic telepresence robot. In Robotics and Automation (ICRA), 2016 IEEE International Conference on, pages 690-695. IEEE, 2016.

[11] Xuan Bo Tran, Nur Hafizah, and Hideki Yanada. Modeling of dynamic friction behaviors of hydraulic cylinders. Mechatronics, 22(1):65-75, 2012.

[12] Saeed Hashemi, Steven Sobojinski, and William K Durfee. Low-friction antagonist hydraulic transmission using long-stroke rolling diaphragm cylinders. In ASME/BATH 2017 Symposium on Fluid Power and Motion Control, pages V001T01A073-V001T01A073. American Society of Mechanical Engineers, 2017.

[13] Stephen Paul Buerger. Stable, high-force, low-impedance robotic actuators for human-interactive machines. $\mathrm{PhD}$ thesis, Massachusetts Institute of Technology, 2005.

[14] Antonia Tzemanaki, Lukasz Fracczak, David Gillatt, Anthony Koupparis, Chris Melhuish, Raj Persad, Edward Rowe, Anthony G Pipe, and Sanja Dogramadzi. Design of a multi-dof cable-driven mechanism of a miniature serial manipulator for robot-assisted minimally invasive surgery. In Biomedical Robotics and Biomechatronics (BioRob), 2016 6th IEEE International Conference on, pages 55-60. IEEE, 2016.

[15] Sotiris Avgousti, Eftychios G Christoforou, Andreas S Panayides, Sotos Voskarides, Cyril Novales, Laurence Nouaille, Constantinos S Pattichis, and Pierre Vieyres. Medical telerobotic systems: current status and future trends. Biomedical engineering online, 15(1):96, 2016.

[16] Adriana Vilchis Gonzales, Philippe Cinquin, Jocelyne Troccaz, Agnès Guerraz, Bernard Hennion, Franck Pellissier, Pierre Thorel, Fabien Courreges, Alain Gourdon, Gérard Poisson, et al. Ter: a system for robotic tele-echography. In International Conference on Medical Image Computing and Computer-Assisted Intervention, pages 326-334. Springer, 2001.

[17] Adriana Vilchis, Jocelyne Troccaz, Philippe Cinquin, Kohji Masuda, and Franck Pellissier. A new robot architecture for tele-echography. IEEE Transactions on Robotics and Automation, 19(5):922-926, 2003.

[18] Thomas Martinelli, Jean-Luc Bosson, Luc Bressollette, Franck Pelissier, Eric Boidard, Jocelyne Troccaz, and Philippe Cinquin. Robot-based teleechography: Clinical evaluation of the ter system in abdominal aortic exploration. Journal of Ultrasound in Medicine, 26(11):1611-1616, 2007.

[19] Farshid Najafi and Nariman Sepehri. A novel hand-controller for remote ultrasound imaging. Mechatronics, 18(10):578-590, 2008.

[20] P. Goldsmith. A fluid-cable transmission for remote actuation, October 24, 2018. US Provisional Patent Application 62/750,131.

[21] A. Schiele. Performance difference of bowden cable relocated and non-relocated master actuators in virtual environment applications. In IEEE/RSJ Internatioinal Conference on intelligent Robots and Systems, pages 3507-3512. IEEE/RSJ, 2008. 